
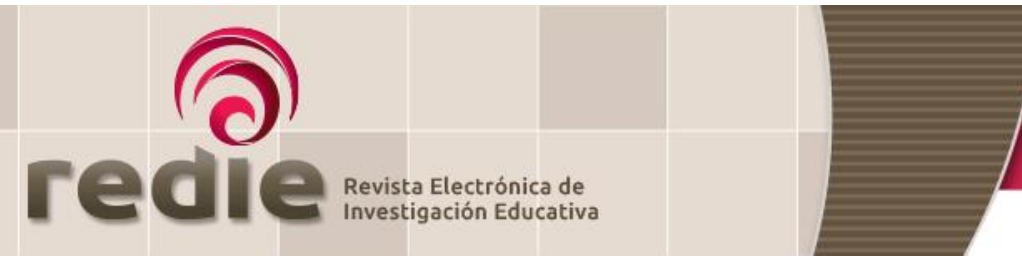

Vol. 19, Núm. 2, 2017

\title{
Análisis descriptivo del gobierno universitario ecuatoriano: una mirada desde los cambios legislativos
}

\section{A Descriptive Analysis of University Administration in Ecuador from the Perspective of Legislative Change}

\author{
Francisco Aníbal Ganga Contreras (1) fagpublicaciones@ulagos.cl \\ Silvia Amir Maluk Uriguen (2) smaluk@espol.edu.ec \\ (1) Universidad de los Lagos \\ (2) Escuela Superior Politécnica del Litoral \\ (Recibido: 25 de agosto de 2014; Aceptado para su publicación: 23 de octubre de 2015)
}

Cómo citar: Ganga, F. A. y Maluk, S. A. (2017). Análisis descriptivo del gobierno universitario ecuatoriano: una mirada desde los cambios legislativos. Revista Electrónica de Investigación Educativa, 19(2), 22-37. https://doi.org/10.24320/redie.2017.19.2.866

\begin{abstract}
Resumen
El sistema universitario ecuatoriano ha experimentado en los últimos años notables transformaciones en el ámbito organizacional. Considerando este escenario, este trabajo tiene como objetivo central describir las modificaciones legislativas más relevantes que afectan el gobierno de las universidades en el Ecuador a raíz de la reforma constitucional de 2008. Para ello se consultaron artículos publicados por autores nacionales y se analizaron los marcos regulatorios más significativos que han regido el funcionamiento de estas Instituciones de Educación Superior (IES). El artículo da cuenta de la carencia de bibliografía científica y especializada en las bases de datos más utilizadas por los académicos. Se plantea, además, la necesidad de generar y potenciar las investigaciones sobre el tema.
\end{abstract}

Palabras clave: Gobierno universitario, educación superior, universidades.

\section{Abstract}

The Ecuadorian university system has undergone significant organizational transformations in recent years. Given this situation, the main objective of this study is to describe the most relevant legislative changes affecting university administration in Ecuador following the 2008 constitutional reform. To this end, this study reviewed papers by Ecuadorian authors and analyzed the most significant regulatory frameworks that have governed the way higher education institutions operate. The paper points out the lack of scientific and specialized bibliography in the most commonly used databases by academics, and makes reference to the need to generate and strengthen research on this topic. 


\section{Introducción}

Las organizaciones experimentan cambios de manera permanente y sistemática, no obstante, los cambios se han intensificado en los últimos años e inciden en todo tipo de instituciones. Las universidades, por supuesto, no quedan al margen de largas, aceleradas, profusas y profundas evoluciones, ante las consecuencias de esto, resulta conveniente analizarlas y estudiarlas.

Considerando el contexto descrito, este trabajo se plantea describir las reformas legislativas más importantes que afectan el gobierno de las universidades en el Ecuador, como consecuencia de la reforma constitucional del 2008. Para ello, se sigue como procedimiento metodológico el uso de fuentes secundarias, es decir, se recurre al escrutinio de artículos académicos, documentos y libros publicados sobre la materia y, por supuesto, se echa una mirada a los aspectos jurídicos más característicos y preponderantes que han regido el funcionamiento de las Instituciones de Educación Superior (IES).

De manera general, desde los años ochenta se evidencia la carencia de estudios que comparen los gobiernos universitarios en Latinoamérica (Levy, 1979), y aunque la bibliografía muestra que el interés por el tema va en aumento, siguen siendo pocas las publicaciones comparativas (Levy, 2006). Las limitaciones metodológicas que pudieran advertirse en el trabajo se corresponden con el hecho de que al ser un marco jurídico reciente, no existen suficientes estudios científicos sobre las IES en el Ecuador bajo el nuevo contexto; si bien el sistema aún está en etapa de transición o adaptación, existen informes en etapa de elaboración que podrían ser de utilidad para futuras investigaciones.

\section{Antecedentes del SES}

Antes de describir algunos cambios del Sistema de Educación Superior (SES) cabe comentar brevemente el desarrollo histórico de las universidades y su modelo regulatorio para comprender el nuevo modelo que ahora es ejecutado en el país por el gobierno en turno.

En general, se reconoce que en el Ecuador el SES ha mantenido una excesiva autonomía durante su acontecer histórico. La regulación gubernamental no ha sido capaz de guiar el desenvolvimiento del mismo para garantizar su solidez y compromiso con la formación profesional del país. Es por esta característica histórica del SES que el Estado ha iniciado el proceso de su transformación, a raíz de la XXI Constitución de la República del año 2008.

Si partimos del concepto de autonomía como un continuo autonomía-heteronomía, en el caso de Ecuador podemos clasificar la autonomía de las universidades como unidimensional: radical frente al Estado y los gobiernos, y totalmente heterónoma frente a los grupos corporativos que existen dentro de las mismas, ante el mercado y grupos económicos y confesionales, grupos de poder de distinta naturaleza, que han puesto diversos intereses en la educación superior (Arcos, 2010, p. 80).

En el pasado hubo intentos de tomar el control del SES por algunos gobiernos en turno; en 1869 el expresidente García Moreno consideró que la Universidad Central del Ecuador (UCE) no formaba profesionales deseables, pues aportaban muy poco al desarrollo Nacional. Por este motivo, y después de un intenso debate, decidió declarar disuelta la UCE para crear -a partir de este hecho histórico- las Escuelas Politécnicas, una nueva figura universitaria con la función de proveer a la nación de técnicos profesionales más especializados en áreas fundamentales para el progreso del país. Para cumplir con esta misión, las Escuelas Politécnicas necesitaban una base académica actualizada, sólida y vinculada a avances tecnológicos de nivel mundial. Para contar con la plataforma requerida, García Moreno acudió al Papa Pío IX y le solicitó el envío de expertos jesuitas que ejercerían las cátedras en las nuevas IES.

Un siglo después, impulsado por la crisis económica del país durante los ochenta, Ecuador implementó las reformas neoliberales conocidas como el "Consenso de Washington", dominantes en América Latina. Una de las características esenciales radicaba en minimizar el tamaño del Estado, desregular los mercados y dejar que se ajustaran a través de su propio mecanismo, buscando dar control a las instituciones privadas. 
En el caso del SES tales reformas "determinaron un alejamiento progresivo del Estado, de su papel regulador y administrador y, consecuentemente, una reducción gradual de los fondos públicos para el financiamiento de las universidades" (Villavicencio, 2012, p. 13). Ante la limitada disponibilidad de fondos y las dificultades económicas de la época fue necesario buscar medios para financiarse de manera privada. El boom petrolero de 1973 fue aprovechado para impulsar la matrícula en las entidades públicas de las primeras urbes del país, como Quito, Guayaquil y Cuenca, desde inicios de los setenta hasta finales de los ochenta (Carvajal, 1990) y de esa manera lograr sobrevivir en un medio neoliberal y competitivo. Hubo que aprender a sobrellevar la situación financiera y convivir con las herramientas del mercado: se amplió la oferta académica por medio de un desproporcionado incremento de las carreras y se adoptaron y promovieron estrategias de captación de alumnos y disminución de costos (Pacheco, 2008).

La educación superior creció un 18.7\% anual entre los años 1974 y 1978, y un 5\% anual en los años 90, durante los cuales las mujeres y los pueblos indígenas tuvieron una creciente participación. El crecimiento de la demanda fue compensado parcialmente por el crecimiento del sector privado. En los 70 había tan solo un par de universidades privadas. Entre 1998 y 2002 el Congreso Nacional estableció la creación de 12 universidades. Hoy en día hay 41 universidades privadas, de las cuales 9 son cofinanciadas por el Estado y 32 son auto-financiadas. Las universidades públicas son 29 de 70 en total. (OEl, 2013).

La tasa neta de asistencia a la educación superior tiene una tendencia al aumento, como se observa en la figura 1. Entre 1982 y 1990, en un período de 8 años creció un 48\%, reflejando el acrecentamiento de la matrícula producto del desarrollo de la producción de petróleo de los años setenta y ochenta. Sin embargo, durante los años noventa este crecimiento disminuyó y se ubicó en un 19\%, posiblemente como muestra de una saturación dada la cantidad de universidades existentes. Luego se observa un retorno al niveles del $53 \%$ en tan solo 5 años, producto del ascenso del poder adquisitivo por la dolarización de la economía en el año 2000, y la creación de 12 nuevas instituciones entre 1998 y 2002.

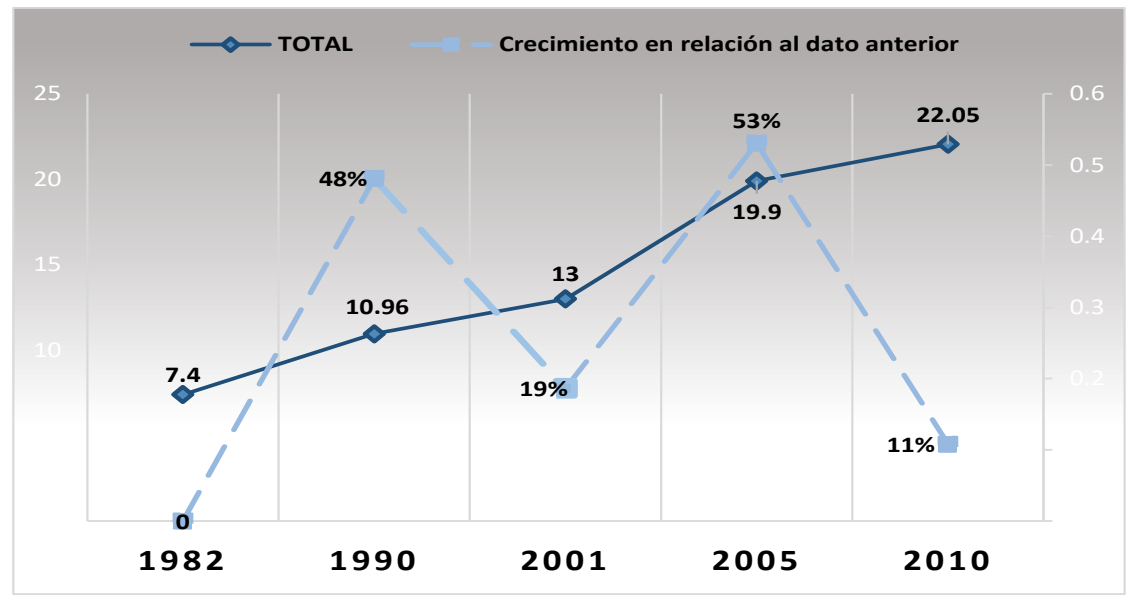

Figura 1. Tasa neta de asistencia en Educación Superior

Fuente: Elaboración propia basado en sIN (2014) y Machasilla et al. (2014).

Para captar un mayor número de alumnos se requería concertar la oferta académica con las profesiones de moda con vistas a satisfacer la visión de la sociedad. Fueron generadas muchas carreras en pocas áreas del conocimiento y se dejaron de lado especialidades que alimentaban al sector productivo, para incrementar otras inherentes al ámbito comercial requeridas en las ciudades más pobladas (Galarza, 2004; Weise y Laguna, 2009). Se abrieron sucursales de pobre infraestructura y baja calidad académica, marcando el inicio de la mercantilización de la enseñanza superior en el Ecuador (Minteguiaga, 2010), legitimada por la visión netamente lucrativa de la Organización Mundial de Comercio, que ve la enseñanza como un servicio financiero. 
Recordemos que en el período 1970-2006 se crearon 56 universidades: 6 a lo largo del ciclo de la reforma de los setenta y 50 durante el período 1982-2006. Es decir, un promedio de dos universidades por año. Entonces los problemas de caos en la oferta académica y titulación y la precarización docente fueron ya detectados, y es en estas circunstancias que la LoEs [Ley Orgánica de Educación Superior] expedida en el año 2000, establece el Sistema Nacional de Evaluación y Acreditación de la Educación Superior bajo la dirección del Consejo Nacional de Evaluación y Acreditación de la Educación Superior (Villavicencio, 2012, p. 11).

En los años noventa, el aún existente Consejo Nacional de Universidades y Escuelas Politécnicas (conuep) realizó un estudio de evaluación de la realidad de las universidades desde el punto de vista institucional, organizativo y académico, lo que se puede considerar como el primer intento de diagnóstico fallido de la educación superior en el Ecuador (CONUEP, 1992). El informe quedó en las gavetas de los escritorios gubernamentales y sólo se pudo acceder a un resumen.

El CONUEP y su "informe oculto" reflejan la débil institucionalidad de los organismos de control. Es verdaderamente penoso que el esfuerzo de más de dos años de 140 profesionales quedara sepultado en 800 páginas que no pudieron contribuir al avance de la academia (Villavicencio, 2012). De igual manera, es lamentable que habiendo existido iniciativas no fueron usadas y pasaran 20 años para ocuparse de una nueva evaluación. Ya en 1992 el documento del cONUEP arrojaba serios problemas (CONUEP, 1992). Se mencionaban los efectos de la crisis de la excelencia académica en el país por más de 20 años. Esto, para Villavicencio (2012) confirma que las IES en el Ecuador podían ser creadas con mucha facilidad y extrema independencia, no integraban un sistema funcional y carecían de sentido académico y curiosidad científica, motivadas únicamente por una finalidad comercial.

En 1998 se reformó nuevamente la Constitución Política del Ecuador y se dio otro intento errado de tomar el control de las IES. Entre las transformaciones propuestas se decidió crear un organismo regulador que reemplazara al CONUEP, que inició sus funciones en el 2000, y el que además de regular las universidades y Escuelas Politécnicas, fiscalizaría a institutos técnicos superiores, tecnológicos, pedagógicos, y conservatorios de música y arte que formaban parte de la educación superior. Esta Constitución reconoce e integra por primera vez el SES en un solo organismo.

Dos años después de la reforma constitucional de 1998 se creó el Consejo Nacional de Evaluación y Acreditación de la Educación Superior (CONEA), cuya función era ejercer la dirección, planificación y coordinación del Sistema Nacional de Evaluación y Acreditación (SNEA), un sistema autónomo e independiente que debía de ser liderado por el CONEA, y coordinado permanentemente con el Consejo Nacional de Educación Superior (CONESUP) para regular y controlar las IES del SES. El nuevo afán tuvo problemas de estructura desde su inicio, una de las principales dificultades radicaba en su composición. Los máximos integrantes eran los rectores de las universidades y de las escuelas politécnicas, o sea, la universidad se regulaba mediante un Consejo cuyo poder estaba centralizado en ella misma. Esto desencadenó una serie de irregularidades y problemas internos que entorpecían las gestiones del CONESUP, pues eran "juez y parte", presentándose el inconveniente conocido como conflicto o problema de doble rol.

Una vez publicada la nueva ley en el año 2000 y establecidas las normas para regular y controlar el SES, las IES generaron resistencia a ser evaluadas. Apelando a la independencia universitaria se negaron a pasar por un sistema de acreditación de calidad, aunque después de unos meses comprendieron que no era negativo tener que cumplir con un requisito formal que validara un nivel de calidad. No obstante las características propias del sistema -basado en procesos limitados y poco claros, se convertiría en un instrumento útil para fortalecer la mercantilización- en un momento dado por el auge económico ocasionado por la dolarización de la economía ecuatoriana "vieron el proceso de evaluación como un mecanismo fácil para ganar legitimidad y posicionarse en el sistema" (Villavicencio, 2012). Se obtuvo provecho del sistema de calidad, que pasó a ser un indicador que facilitó la comercialización de las carreras. 
El informe del CONEA para la UNESCO/IESALC, que recogía los antecedentes, situación y perspectivas de la evaluación y la acreditación de la educación superior en 2003, indica el grado de conocimiento sobre el estado de las IES en ese momento y constituye una prueba más de la necesidad de generar reflexión en el mundo universitario. Se aborda el tema de la acreditación superior en el Ecuador como algo novedoso en Latinoamérica y se presenta el Perfil del Plan de Desarrollo de las Universidades y Escuelas Politécnicas (PLANUEP), con el cual se intentaba posicionar estratégicamente a la universidad en la sociedad, además de idear un nuevo sistema de acreditación. Esta iniciativa se fundamentaba en:

Cinco "nudos críticos" que la universidad ecuatoriana debía de resolver para romper su estancamiento y enfrentar los desafíos del nuevo milenio: Insuficiente vinculación de las Universidades y Escuelas Politécnicas con el medio externo; Insuficiente calidad de la actividad académica universitaria; Baja calidad de la gestión; Insuficiencia de recursos económicos y; Carencia de un sistema de rendición social de cuentas. (Consejo Nacional de Evaluación y Acreditación de la Educación Superior, 2003, p. 2).

La escasa funcionalidad del SES refleja una vez más la débil institucionalidad de los organismos de regulación del gobierno ecuatoriano. Una tentativa tras otra de tomar el control del sistema universitario demuestra la falta de políticas públicas definidas y orientadas al desarrollo de las universidades, carentes además de un sistema dotado de forma y sinergia, "lo que acarrea consecuencias negativas incalculables para la excelencia académica" (Villavicencio 2012, p. 10).

Fundamentado en lo anterior el Estado decidió retomar el protagonismo en el direccionamiento de la educación superior a través de una reforma constitucional que condujo a importantes cambios en la forma de gobernar, regular y controlar las IES.

\section{Cambios en el gobierno universitario}

El SES está conformado por 29 Institutos Superiores Pedagógicos e Inter-bilingües, 15 Institutos Superiores de Arte y Conservatorios Superiores, 221 Institutos Superiores Técnicos y Tecnológicos, 50 Universidades y 5 Escuelas Politécnicas (Consejo de Educación Superior 2015), un total de 320 organizaciones involucradas en la formación de los ciudadanos ecuatorianos en el tercero y cuarto nivel.

Siguiendo a Schwartzman y Klein (1994) y Levy (2006), las IES en el país andino se pueden tipificar, además de por el origen de propiedad y su oferta académica, por su tipo de financiamiento, la formación del profesorado y la ubicación geográfica. Las instituciones públicas suman 28, las privadas 20 y las promovidas y patrocinadas por congregaciones religiosas son 6.

También existen en el país dos universidades con iniciación supranacional: la Facultad Latinoamericana de Ciencias Sociales (FLACSO) y la Universidad Andina Simón Bolívar (UASB), ambas con sedes regionales. Su propiedad se suscita a través de los parlamentos y consejos administrativos establecidos por tratados internacionales. La FLACSO es una iniciativa de la UNESCO de apoyo a la comunidad latina en pos de abrir espacios de reflexión y debate, mientras que la UASB es obra de Comunidad Andina de Naciones (CAN), forma parte de su sistema de integración y ejerce un papel fundamental como centro académico cultural y científico. Son entidades donde se imparten exclusivamente cursos de posgrado (CEAACES, 2012).

En cuanto al financiamiento, un 50\% de las universidades lo obtienen del Estado como respuesta a la gratuidad de la educación pública desde la Constitución del 2008. Por otra parte, coexisten un $14 \%$ de instituciones particulares, las que mantienen cierto grado de autonomía en sus operaciones pero también reciben rentas estatales en un porcentaje determinado por su nivel de calidad y la cifra de alumnos que acogen, entre otras razones. El 32\% recae en las IES autofinanciadas, capitalizadas en su totalidad por aranceles estudiantiles y fuentes privadas, estas IES poseen un mayor grado de independencia económica. Las supranacionales cuentan con recursos de países miembros de los tratados internacionales que las originan (Consejo de Educación Superior, 2015). 
En relación a las áreas del conocimiento, según datos del CEAACES (2012), más del 40\% de los matriculados estudia carreas relacionadas con las Ciencias Sociales, la Enseñanza Comercial y el Derecho, la Educación concentra casi el 20\%, mientras que áreas destinadas a la Ingeniería, la Industria y Construcción representan el 12\%. Para cubrir esta oferta académica se cuenta únicamente con un 3\% de los docentes con grado de doctor, mientras un 70\% tiene un nivel de formación de diplomados y magister.

Desde el punto de vista geográfico, prácticamente un 30\% de las universidades en el Ecuador se concentran en Quito, su capital política. Guayaquil y sus alrededores, Milagro y Samborondón superan levemente el 20\%, y ciudades vecinas de la sierra como Ambato y Riobamba un 9\%. Cuenca, conocida por su desarrollo cultural, cuenta sólo con un 7\%, las localidades linderas de Manta y Portoviejo con un 5\%, y Loja en el centro-sur de la nación con un $4 \%$. El resto de las IES equivale a un $26 \%$, repartidas en los demás distritos del país (SENESCYT, 2013).

La educación superior en el Ecuador, a partir de la Constitución de 2008, está dirigida bajo los lineamientos del "Buen Vivir", considerado por el gobierno como el estándar de vida deseable para el país y con el cual se busca crear una "universidad diferente para una sociedad diferente" (Ramírez, 2010). Con este modelo se introduce la Recuperación de lo Público en relación a la Universidad y su misión. Para materializar tal desafío, desde el 2008 la educación superior es apreciada como un "bien público". De esta manera, se establecieron exigencias claves en los artículos relacionados con la temática en aras de fraguar una educación de calidad para el "Buen Vivir" de todos los ecuatorianos, en armonía con la naturaleza.

En la Constitución, específicamente en el Art. 28, el capítulo de los Derechos del Buen Vivir establece que la educación superior responderá al interés público y no estará al servicio de intereses individuales y corporativos. Con ello se busca que la res publica o "cosa pública" recupere el protagonismo en un sector trascendente para el desarrollo social, encauzado hacia una "sociedad que aprende".

La Constitución de 2008 define al SES como una herramienta estratégica para la formación de la sociedad orientada al avance científico y profesional: un espacio de intercambio cultural y conservación de los saberes ancestrales. Se ve a la universidad como hacedora del avance científico, encargada de dar respuesta a los problemas del país, concernientes a los lineamientos del Plan Nacional del Buen Vivir del Sumak Kawsay, el que a su vez se nutre de buena educación y comunicación, garantiza la transmisión de saberes y valores de generación en generación y su articulación con otros conocimientos. La educación ha de estar en función de la sociedad y de los intereses comunes y ha de ser una educación que valore los saberes de todas las culturas (Chuji, 2010, p. 231). Textualmente, la normativa establece:

El sistema de educación superior tiene como finalidad la formación académica y profesional con visión científica y humanista; la investigación científica y tecnológica; la innovación, promoción, desarrollo y difusión de los saberes y las culturas; la construcción de soluciones para los problemas del país, en relación con los objetivos del régimen de desarrollo. (Asamblea Constituyente, 2008, p. 350).

El propósito del SES es recuperar el rol protagónico del Estado en la educación superior para lograr, además de la calidad, la regulación de la actividad y la inclusión de todos los factores respetando la pluriculturalidad y la equidad mediante políticas de acción afirmativa. Por medio de las cuales se busca una plataforma común fundadora de conocimientos, con sólidos principios democráticos, donde no haya distinción de clases o grupos étnicos.

No obstante, la debilidad histórica de la universidad ecuatoriana ha radicado principalmente en la vulnerabilidad de sus políticas académicas. Durante décadas la capacidad del Estado de implementar políticas públicas eficaces, legítimas, íntegras, participativas y transparentes ha sido muy débil y poco realista. La gobernanza de la evaluación y acreditación muestra la escasez de principios claros y definidos, capaces de apoyar el fortalecimiento tanto de las IES como del SES en su totalidad (Pacheco, 2011; Villavicencio, 2012).

Las estrategias que ahora están siendo implementadas no son nuevas; el cierre de universidades, 
regulación de la oferta académica, y generación e importación de académicos investigadores, en áreas correspondientes a las líneas del Plan de Nacional de Desarrollo (PND) del gobierno, ya han sido prácticas que gobiernos anteriores han intentado utilizar para tomar el control de la educación superior. En 1869, la clausura de la Universidad Central del Ecuador (UCE), la creación de las escuelas politécnicas y la estrategia de importación de expertos académicos jesuitas en temas de gran importancia para el desarrollo del país, fueron intentos gubernamentales para transformar la universidad en el Ecuador. Esta estrategia política para mejorar las bases técnicas de las escuelas politécnicas Villavicencio (2012) la compara con la estrategia gubernamental actual del programa PROMETEO'.

Estas estrategias van en el sentido de la generación de una homogenización de la educación superior y requiere de tres factores:

1) imitación institucional, un proceso mediante el cual las de menor calidad intentan imitar a las de mayor calidad; 2) el cambio de educación privada a educación pública, y 3) el traslado de la principal corriente de instituciones que han servido a clientes especializados, abriendo sus puertas a un espectro aún mayor de estudiantes (Baldridge, 1977).

\section{Cambio de paradigma de los organismos que controlan las SES}

Tanto el CONESUP como el CONEA resultaron ser entidades débiles, apenas sin capacidades para asentar sus políticas, ejercer presión sobre las IES y conducirlas a niveles de acreditación y altos estándares de calidad. El CONEA no consiguió legitimarse, ni siquiera posicionarse dentro del SES dada la carencia de asignaciones presupuestarias, la falta de un marco regulatorio fuerte, la ausencia de normativas claves para el funcionamiento de los organismos de control y la implementación de procesos engorrosos, caros y extensos (Villavicencio, 2012).

Por consiguiente, hubo consecuencias negativas que recayeron sobre la credibilidad del SES ecuatoriano. Las universidades se manejaban según sus propios preceptos siguiendo la norma de "no dejar hacer" nada que pudiera restarles protagonismo dentro del espectro de la educación superior, convirtiéndose en el eslabón más débil de una cadena básica para el progreso nacional. Esta pobre capacidad de acción ocasionó un incremento desproporcionado e injustificado de la oferta académica (Villavicencio, 2012).

Como lo analiza Minteguiaga (2010), "los regulados eran los reguladores"; el autor describe un caso claro de la manipulación del organismo "controlador" por parte de la comunidad universitaria. El conesup, al estar sometido a las universidades, durante sus diez años de vigencia posibilitó que las IES estuvieran prácticamente libres y sin rumbo, con absoluta autonomía pero abrumadas por las presiones del mercado.

Durante este período las entidades se autorregulaban; el CONESUP era una especie de "Asamblea de Rectores" en la que se tomaban decisiones fundamentadas en los intereses particulares de cada universidad y se generaban debates conflictivos en torno a las distribuciones de recursos y los cupos. Se desconocía prácticamente la misión fundamental de la universidad como parte de la sociedad; no rendían cuentas a nadie, solo a sí mismas (Minteguiaga, 2010; Villavicencio, 2012).

En realidad esta breve narración de acontecimientos, que parecen a simple vista erráticos y contradictorios, devela que el problema de fondo parece estar en el diseño institucional y en la lógica política que asume un organismo que es al mismo tiempo juez y parte; es decir, un organismo que debe regular al campo pero que está integrado por los propios regulados; un organismo que debe velar por el cumplimiento de la ley, pero simultáneamente sus miembros pueden tener conflicto de intereses con su aplicación estricta (Minteguiaga, 2010).

1 PROMETEO es una iniciativa que busca promover y fortalecer la educación, la investigación científica y el desarrollo tecnológico en todas las áreas estratégicas de desarrollo del Ecuador, mediante la contratación de docentes investigadores que residen en el extranjero y que tengan un título PhD, quienes trabajarán conjuntamente con una institución anfitriona en un proyecto de investigación. 
Es por esto que según Arcos (2010) no deja de sorprender que uno de los argumentos más recurridos durante el debate de la nueva LOES sea la autonomía universitaria. Un concepto que ante la amenaza de quitarle el privilegio de la "autorregulación" a la Universidad ecuatoriana, logró unir vertientes de ideologías que por décadas han estado en bandos contrarios. Unos se dicen de izquierda y otros de derecha, pero esta vez, ante los contundentes cambios propuestos en los gobiernos de los organismos de regulación del SES, se generó un intenso debate sobre la autonomía universitaria en el país, y "no deja de ser una paradoja que trayectorias políticas, intelectuales e institucionales distintas se conjugan en el uso del concepto autonomía como hito, barrera, trinchera de la defensa de la universidad frente al envite de una reforma" (Arcos, 2010, p. 58). La universidad ecuatoriana se unió en defensa de sus intereses y marchó de la mano como un solo cuerpo por las calles del país, con una participación activa nunca antes vista en la historia de estas instituciones.

La figura 2 muestra cómo las IES en la LOES del año 2000 tenían una relación bastante estrecha con los organismos de control externo. Las IES bajo este esquema regulatorio ejercían un doble rol; los rectores y delegados eran los miembros de los máximos cuerpos administrativos de los organismos reguladores del SES. Entonces, los organismos de control como la Asamblea de Universidades del Ecuador (AUE), el cONESUP y CONEA carecen de institucionalidad y se convierten en simples figuras burocráticas que poco podían hacer para orientar al SES ecuatoriano (Minteguiaga, 2010; Villavicencio, 2012).

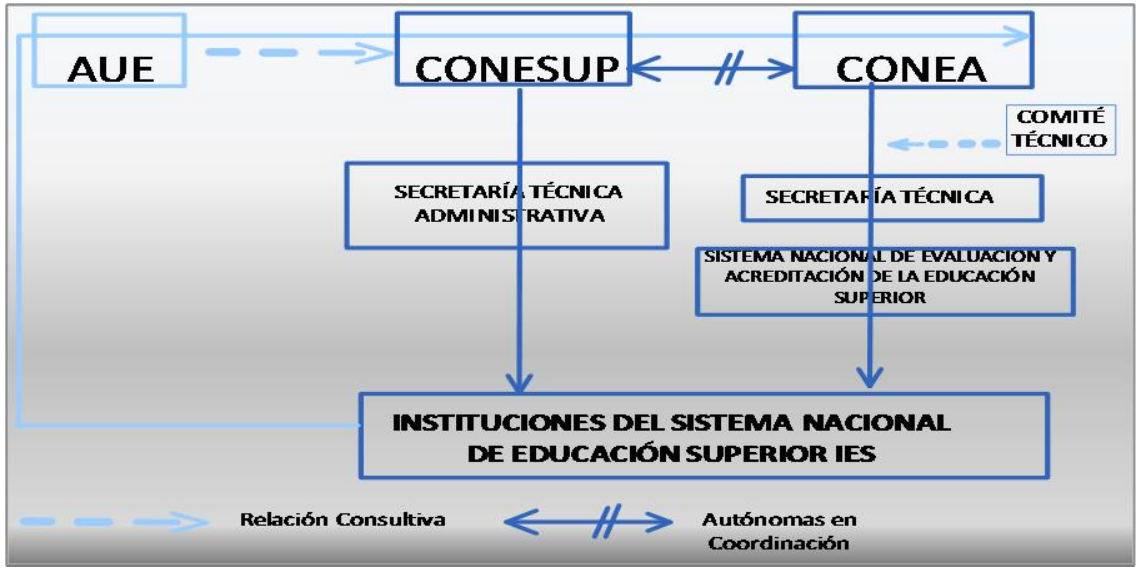

Figura 2. Organismos de Control del Sistema Nacional de Educación Superior hasta el 2008

Fuente: Elaboración propia basado en LOES (CNE, 2000).

Este defecto llevó a que uno de los cambios más significativos en la LoES del año 2010 estableciera justamente en el gobierno los organismos de control, desde las jerarquías organizacionales y dependencias institucionales hasta la conformación de los consejos rectores y sus funciones. La universidad pasó de "regularse a sí misma" a ser un órgano asesor de los organismos de control y regulación y, por consiguiente, al cambiar la manera de regular y de controlar el sistema desde afuera, se modifica la forma de gobernanza de las universidades desde adentro (Ramírez, 2013).

De esta manera se llegó a un modelo en el cual la universidad no consigue regularse ni participar de su propia regulación. Los máximos cuerpos de gobierno de los organismos de control no pueden ser integrados por ningún funcionario que sea parte activa de la institución. Se impide que la propia entidad influya directamente sobre el proceso regulatorio, ésta fungirá sólo como órgano consultor de los organismos de control mediante asamblea. Parte importante de la conformación de los cuerpos de gobierno de los organismos de control la dispone el ejecutivo. 
Es de interés del Estado tomar las riendas de la regulación de la educación superior y tener una participación activa en este proceso, acción vista por el gobierno a través de los académicos y funcionarios delegados por el Presidente de la República, en el gobierno de los organismos de control.

Por estas razones, una de las disputas políticas más relevantes que se enfrentaron a partir de 2008 está relacionada, por un lado, con des-corporativizar a los órganos rectores del sistema y, por otro, con la creación de una Secretaría de Estado dentro del Poder Ejecutivo nacional, cuya función consistiera en hacer política pública para el campo de la educación superior y para el complejo científico-tecnológico nacional.

En efecto, luego de aprobada por referendo popular la Constitución de la República y de la expedición en 2010 -bajo las disposiciones del nuevo marco constitucional- de una novel Ley Orgánica de Educación Superior, se crea la Secretaría Nacional de Educación Superior, Ciencia, Tecnología e Innovación (SENESCYT), encargada de elaborar y coordinar políticas públicas para el sistema y articularlo con el sector.

Asimismo, se establece el Consejo de Educación Superior (CES) y el Consejo de Evaluación, Acreditación y Aseguramiento de la Calidad de la Educación Superior (CEAACES). Ambos órganos colegiados asumen una composición mixta. El CES está integrado por seis académicos y un estudiante - todos nombrados por el Consejo Nacional Electoral (CNE) a través de un concurso público de merecimiento y oposición-, y por cuatro ministros de Estado.

A su vez, el CEAACES está conformado por seis miembros, de los cuales tres son académicos delegados por el Presidente de la República y tres académicos designados por concurso público de merecimiento y oposición por el mismo CNE. Todos deben cumplir los mismos requisitos de una rectora o rector universitario; sin embargo, no pueden ser autoridades universitarias. En la misma línea de transformaciones, la Asamblea Universitaria, recobrando su espíritu original, queda integrada por los distintos estamentos de su comunidad (rectores, estudiantes, académicos y trabajadores), y asume importantes funciones de asesoría a los órganos gubernativos del sistema de educación superior. (Ramírez, 2013, p. 25).

Los Organismos de Regulación y Control del SES, y los cuerpos de gobierno que los conforman, están normados en la LOES del 2010 (Asamblea Nacional, 2010, cap. 2): Consejo de Educación Superior (CES), Consejo de Evaluación, Acreditación Y Aseguramiento de la Calidad de la Educación Superior (CEAACES), Asamblea del SES (ASES) Órgano Consultivo, Secretaría Nacional de Educación Superior, Ciencia, Tecnología e Innovación (SENESCYT) y Comités Regionales Consultivos de Planificación de la Educación Superior.

Consejo de Educación Superior (CES): Tiene por objetivo la planificación, regulación y coordinación interna del SES y la relación entre sus distintos actores con la Función Ejecutiva y la sociedad ecuatoriana; está integrado por:

a) Cuatro representantes del Ejecutivo:

i. Secretario Nacional de Educación Superior, Ciencia Tecnología e Innovación.

ii. Secretario Técnico del Sistema Nacional de Planificación o su delegado.

iii. Ministro que dirija el Sistema Educativo Nacional o su delegado.

iv. Ministro que dirija la Política de Producción o su delegado.

b) Seis académicos elegidos por concurso público de merecimientos y oposición:

i. Deberán cumplir los mismos requisitos necesarios para ser Rector universitario o politécnico.

ii. Ser electos por los siguientes criterios: áreas de conocimiento, equilibrio territorial y de género. No podrán posesionarse las autoridades académicas y administrativas de los organismos o instituciones objeto del control y regulación del sistema. 
c) Un representante de los estudiantes que participará en las sesiones con derecho a voz.

Consejo de Evaluación, Acreditación y Aseguramiento de la Calidad de la Educación Superior (CEAACES): Normará la autoevaluación institucional y ejecutará los procesos de evaluación externa, acreditación, clasificación académica y el aseguramiento de la calidad. Este organismo estará compuesto por:

a) Tres seleccionados por concurso público de méritos y oposición organizado por el Consejo Nacional Electoral, quienes cumplirán los mismos requisitos dispuestos para ser Rector de una universidad.

b) Tres designados por el Presidente de la República.

Asamblea del SES (ASES) Órgano Consultivo: Órgano representativo y consultivo que sugiere al Consejo de Educación Superior, políticas y lineamientos para las instituciones que conforman el SES. Con fines informativos, conocerá los resultados de la gestión anual del Consejo. Estará integrado por:

a) Todos los rectores de las universidades y escuelas politécnicas públicas y particulares que integran el SES.

b) Un profesor titular principal elegido mediante votación secreta y universal por cada universidad y escuela politécnica pública.

c) Dos por las universidades y escuelas politécnicas particulares.

d) Seis representantes de las y los estudiantes.

e) Ocho rectores representantes de los institutos superiores.

f) Dos representantes de las y los servidores y las y los trabajadores universitarios y politécnicos del Ecuador.

En la conformación de la Asamblea se garantizará la equidad, alternancia y la paridad de la representación entre hombres y mujeres.

Secretaría Nacional de Educación Superior, Ciencia, Tecnología e Innovación (SENESCYT): Órgano que tiene por objeto ejercer la rectoría de la política pública de educación superior y coordinar acciones entre la Función Ejecutiva y las instituciones del SES. Este organismo está dirigido por el Secretario Nacional de Educación Superior, Ciencia, Tecnología e Innovación de Educación Superior, designado por el Presidente de la República.

Comités Regionales Consultivos de Planificación de la Educación Superior: Órganos de consulta regional de la Secretaría Nacional de Educación Superior, Ciencia, Tecnología e Innovación, de articulación con el trabajo desconcentrado de la Función Ejecutiva y de coordinación territorial con los actores de la educación superior que trabajen a escala regional y de los gobiernos regionales autónomos. Su finalidad es constituirse en herramienta de consulta horizontal del SES a nivel regional. La integración de cada uno de estos comités será normada en el instructivo que para el efecto expida la Secretaría Nacional de Educación Superior, Ciencia, Tecnología e Innovación.

La figura 3 representa los organismos previamente definidos. En el nuevo esquema del modelo del SES, se observa que ya las IES no forman parte de los cuerpos administrativos de los organismos de control, sino que han pasado a conformar una asamblea asesora. La relación ya no es gerencial, ahora es consultiva entre las IES y la regulación. 


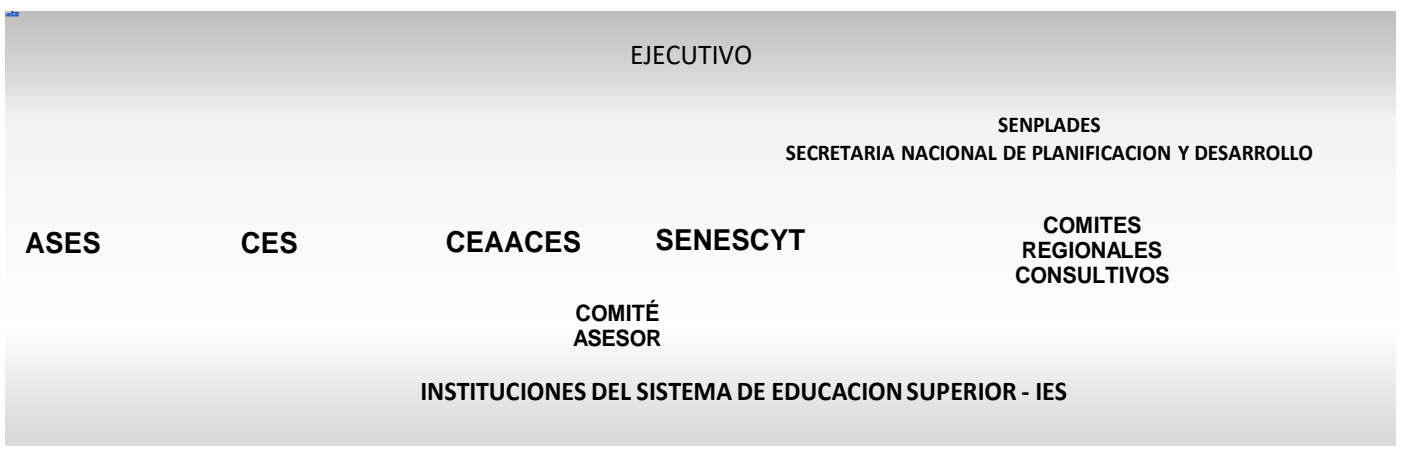

Figura 3. Organismos de Control del Sistema Nacional de Educación Superior a partir del año 2008

Fuente: Elaboración propia basado en Asamblea Constituyente (2008) y Asamblea Nacional (2010).

\section{Cambios que afectan la gobernanza universitaria}

La reforma del modelo universitario empezó a ejecutarse en el 2008 con el mandato Presidencial número 14, que dio inicio a otra valoración de las universidades a través de los organismos competentes y permitió restablecer indicadores sobre el estado del sistema con el fin de categorizar a las IES según su nivel de calidad. El CONEA, organismo que mantenía su competencia en 2008, emprende la evaluación con un informe denominado Evaluación de Desempeño Institucional de las Universidades y Escuelas Politécnicas del Ecuador.

El documento logró sintetizar lo que se sabía desde hacía 20 años, la necesidad de realizar nuevamente una estimación capaz de instruir formalmente la etapa de depuración del SES: "Ante todo, el Informe constituyó el primer paso de un proceso de recuperación del Estado de su rol de dirección, regulación y supervisión de un bien público como es la educación superior" (Villavicencio, 2012, p. 9). Éste evidenció la urgencia de una restauración del sistema universitario enfocada en su reorientación hacia una visión cultural y de generación de conocimientos, como centro promotor de ideas y debates deliberantes y con una decisiva participación social de todos los actores de la comunidad. Posibilitó la creación de conciencia sobre el estado del SES, y el resurgimiento de expectativas positivas respecto a la calidad de las IES.

El Art. 351 de la Constitución política del 2008 establece los cambios que afectarán directamente al gobierno corporativo: El SES se regirá por los principios de Autonomía Responsable y Cogobierno. No se elimina a la Autonomía Universitaria, aunque en la práctica se verá bastante limitada dadas las crecientes exigencias de los organismos de control. Se percibe además la necesidad de las universidades de formar parte de la lista que distingue a las instituciones con buenas calificaciones respecto a la calidad. Los cambios bien se podrían asociar como de control estatal, no de supervisión, descritos por Levy (2006), "demuestran centralización, planeamiento gubernamental a priori, fuerte control gubernamental e imposiciones estatales estandarizadas a lo largo de las instituciones".

Cuando de control estatal se trata, Levy (1979) recomienda profundizar sobre el entendimiento de las relaciones entre el tipo de régimen y el nivel de desarrollo nacional, con el grado de autonomía universitaria. En sus publicaciones no se han encontrado correlaciones significativas entre estos factores, situación que ha sido muy poco explorada en la educación superior. En este sentido, y tomando en cuenta que el cogobierno es parte ineludible de una autonomía universitaria responsable, y ésta es determinada por quienes representan los Órganos Colegiados de carácter académico y administrativo junto a las unidades de apoyo; todo ello gravita en una dirección compartida de las universidades y escuelas politécnicas por parte de los diferentes sectores de sus comunidades: profesores, estudiantes, empleados, trabajadores, y ex alumnos. El cogobierno debe de conformarse respetando los principios que rigen en el SES, establecidos en la Constitución y en la Ley: calidad, igualdad de oportunidades, alternabilidad y equidad de género. 
En primer lugar, la recuperación de lo público está relacionada con la consciencia del impacto que produce el bien educación superior, el cual afectará tarde o temprano a la sociedad en su conjunto, sin importar si es gestionado por actores estatales o particulares. [...] En segundo lugar, lo público está relacionado con la deselitización del campo universitario; o, dicho de otro modo, con la democratización en el acceso, tránsito y egreso de la universidad, y también con la democratización de la toma de decisiones al interior de los centros de estudio (cogobierno). (Ramírez, 2013, p. 15).

\subsection{Enfoque y redireccionamiento de las IES}

Integran el SES 477 IES, de las cuales 407 son Institutos Tecnológicos, Pedagógicos y Conservatorios de Arte, mientras que el resto son Universidades y Escuelas Politécnicas. Los niveles y tipos de IES en el Ecuador están normados por la LOES en su Art. 117; y se definen de acuerdo a las actividades académicas que cumplen: instituciones de docencia con investigación, instituciones orientadas a la docencia e instituciones dedicadas a la enseñanza superior continua. Los elementos más relevantes son:

- El Estado garantizará el financiamiento de las instituciones públicas de educación superior.

- Las IES públicas serán gratuitas hasta el 3er. nivel.

- La distribución de recursos se basará en la calidad y criterios definidos en la ley.

- La ley regulará las fuentes alternativas de ingresos para las IES públicas y particulares.

- Los profesores son servidores públicos sujetos a un régimen propio que estará contemplado en el Reglamento de Carrera.

- En las IES particulares se observarán las disposiciones del Código de Trabajo.

- Las universidades y su oferta académica deben de estar alineadas con el Plan de desarrollo Nacional.

- El Plan Nacional del Buen Vivir, planteado por el gobierno ecuatoriano, establece la necesidad de transformar la universidad para cambiar la sociedad. En la medida en que el conocimiento sea un medio para radicalizar la democracia, generar una nueva forma de acumulación sostenible ambientalmente y edificar relaciones sociales no capitalistas, podrá constituirse en el motor de la construcción de una sociedad emancipada (Ramírez, 2010, p. 15).

- Se tipifican las IES en función del ámbito de las actividades académicas que realicen, existiendo por lo tanto, instituciones de docencia con investigación, instituciones orientadas a la docencia e Instituciones dedicadas a la educación superior continua.

- Constituye obligación de las instituciones del SES, la articulación con los parámetros que señale el Plan Nacional de Desarrollo y que al menos un $70 \%$ de los títulos otorgados por las escuelas politécnicas deberán corresponder a títulos profesionales en ciencias básicas y aplicadas.

Con respecto al cambio de autofinanciamiento a la gratuidad, hay quienes respaldan ambas vertientes, no obstante, aún es pronto para determinar sobre cuál modelo es más eficaz en el cumplimiento de los objetivos sociales planteados por el Sumak Kawsay. El principal argumento para defender la gratuidad es su contribución al principio de equidad e igualdad social y su incidencia en que personas, que por décadas han sido marginadas del ingreso a la universidad por razones económicas, puedan acceder a una educación pública de calidad, vivir dignamente, y dejar atrás las élites económicas y empezar a tener ciudadanos intelectuales (Ramírez, 2010).

Otros autores argumentan que las buenas intenciones sociales y los ideales políticos, no pueden ser fácilmente traducidos en realidad, ya que a veces "la medicina puede ser más grave que la enfermedad" al desarrollar políticas públicas que ocasionen efectos contraproducentes en la situación educativa del país. Post (2011) recomienda no perderle la pista a las consecuencias, y aunque aún es prematuro para sacar conclusiones sobre los efectos de la gratuidad en la equidad social, cabe señalar que el peso financiero del gasto público en educación superior esté dejando de lado reformas indispensables en la educación primaria y secundaria que contribuirían a que el cambio en el acceso a la formación terciaria sea más realista. 
Por otro lado, en lo que respecta al cambio en la orientación de las IES, en la figura 4 es factible observar esquemáticamente una síntesis del SES.

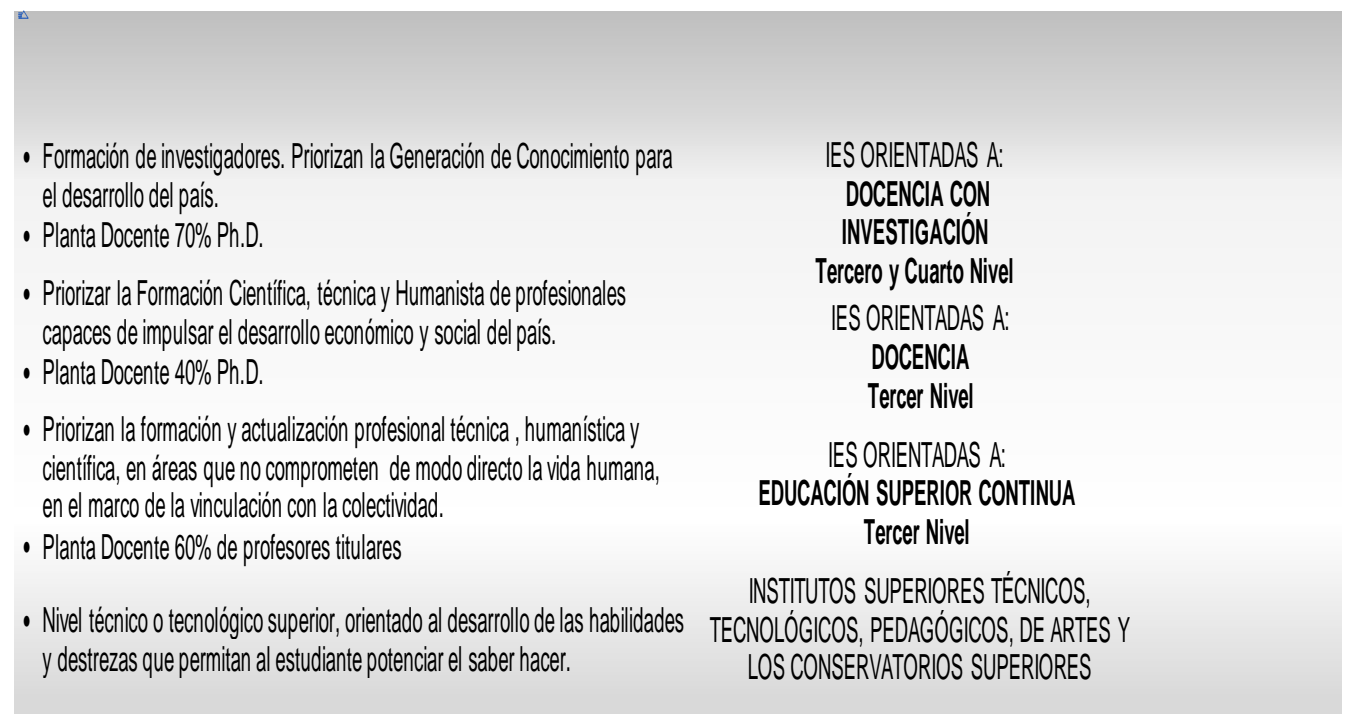

Figura 4. Niveles y tipos de universidades en el Ecuador

Fuente: Elaboración propia basado en Ganga (2005), Asamblea Nacional, (2010) y CEAACES (2013).

Como se observa, sólo aquellas universidades que tenga un 70\% de planta pedagógica con PhD pueden ser calificadas como de "Docencia con Investigación" y eso determinará el tipo de financiamiento estatal que recibirán para proyectos, programas de doctorado o estar en condiciones de realizar llamados a concursos públicos para la contratación de profesores con mayor experiencia según el último Reglamento de escalafón docente. Actualmente ninguna universidad del Ecuador cuenta con la categoría "de investigación", pues sus plantas aún están en proceso de formación.

\section{Conclusiones}

Los cambios detallados en el presente trabajo se dividieron en cuatro aspectos principales: los que afectan a la administración universitaria, las modificaciones en el paradigma del gobierno de los organismos que inciden en la regulación y el control del SES, las transformaciones en la conformación de los gobiernos corporativos universitarios que apuntan directamente la gobernanza, y el enfoque y redireccionamiento de las IES en cuanto a su financiamiento, tipología y orientación académica, los cuales han sido posibles gracias al incremento de la institucionalidad de los organismos de regulación y control del SES. Mediante la reestructuración del gobierno corporativo se consiguió atenuar el inconveniente del doble rol que las universidades ejercían como juez y parte de la regulación. Los cambios aludidos reflejan la intención de centralizar en el gobierno y de visualizar a través de éste la orientación y la producción científica y profesional.

Se advirtió, además, cómo algunos de los autores sugieren que la intención del modelo es recobrar el papel protagonista del estado en la regulación y control del SES.

¿Hasta dónde llega el protagonismo y donde empieza la autonomía? ¿Qué papel jugará la universidad dentro de las reformas? ¿Se dejarán llevar por el modelo gubernamental o generarán investigación científica sobre sí misma para construir otro más cercano al contexto cultural y a la realidad académica del país? Son algunas de las interrogantes que plantea este artículo y que sería recomendable responder para lograr conocimientos sobre y para la universidad ecuatoriana. 
Una de las principales limitaciones que enfrentó el estudio fue la falta de publicaciones sobre el SES en el Ecuador, además de que las reformas están en proceso de elaboración. La mayoría de las fuentes utilizadas corresponde a libros o revistas nacionales, y a opiniones de poca rigurosidad. Lamentablemente, después de realizar una amplia búsqueda en bases de datos, coincidimos en que en el Ecuador no se han generado investigaciones sobre el sistema universitario.

El ambiente organizacional y político referido evidencia que es a partir de las reformas constitucionales del 2008 que se intenta retomar el diálogo y el análisis de la Universidad y su sistema en el país andino. Precisamente es a causa de este autoabandono que la principal restricción para desarrollar el trabajo coincide con que en el Ecuador no se haya pensado en el tema por más de 20 años.

Otro de los aspectos adversos para la investigación apunta a que las reformas son recientes y los instrumentos legales se hallan en un proceso de actualización. Aún hay ciertos vacíos, reglamentos por elaborar, estatutos universitarios estancados o en proceso de diálogo y aprobación.

Por otro lado, los académicos están a la espera de la disponibilidad de las bases de datos estadísticas recaudadas durante los procesos de evaluación y acreditación del 2010 al 2013; dado que éstas servirán para complementar las indagaciones que están llevando a cabo. Esta condición de transitoriedad en el contexto jurídico y en otros aspectos universitarios que aún se desconocen afectan el contexto organizacional y pueden tener efectos sobre el gobierno universitario del SES ecuatoriano. Es por esta condición, producto del hecho de que aún están vigentes los tiempos de transición entre los contextos jurídicos y normativos, que este trabajo no describe el producto final del nuevo SES.

Como ha quedado de manifiesto, abordamos una temática desafiante, pero que abre nuevas vías investigativas, especialmente pensando en los efectos que se generarán, así como por las opciones que representa realizar estudios comparativos, tanto a nivel latinoamericano como iberoamericano, pero también en el ámbito global.

\section{Referencias}

Arcos, C. (2010). La autonomía como argumento político. En R. Ramírez (Ed.), Transformar la Universidad para transformar la sociedad (pp. 57-82). Quito: SENPLADES.

Asamblea Constituyente. (2008). Vigésima Primera Constitución de la República del Ecuador.

Asamblea Nacional. (2010). Ley Orgánica de Educación Superior. Registro Oficial No. 298.

Baldridge, J. V. (1977). Diversity in higher education: professional autonomy. The Journal of Higher Education, 48, 367-388.

Carvajal, I. (1990). La universidad ecuatoriana y su perspectiva en los 90. En CONUEP (Ed.) La universidad ecuatoriana y el desarrollo nacional (pp. 141-185). Quito: Consejo Nacional de Universidades y Escuelas Politécnicas.

Consejo de Evaluación, Acreditación y Aseguramiento de la Calidad de la Educación Superior. (2012). Información del Sistema Nacional de Información de la Educación Superior. Quito.

Consejo de Evaluación, Acreditación y Aseguramiento de la Calidad de la Educación Superior. (23 de septiembre de 2013). Reglamento Transitorio para la Tipología de Universidades y Escuelas Politécnicas y de los tipos de carreras o programas que podrán efectuar cada una de estas instituciones. Recuperado de http://www.ceaaces.gob.ec/images/stories/documentacion/normativas/reglamento\%20transitorio\%20p ara\%20tipologa\%20de\%20universidades.pdf 
Chuji, M. (2010). Sumak Kawsay versus desarrollo. En A. L. Hidalgo-Capitan, A. Guillén y N. Deleg (Eds.), Antología del pensamiento indigenista ecuatoriano sobre el Sumak Kawsay. España: FIUCUHU.

Consejo Nacional de Evaluación y Acreditación de la Educación Superior (2003). Los antecedentes, situación actual y perspectivas de la evaluación y acreditación de la educación superior. Quito: UNESCO/IESALC.

Consejo Nacional de Evaluación y Acreditación de la Educación Superior. (2009). Informe de Evaluación de la Educación Superior. Quito: Autor.

Congreso Nacional del Ecuador. (15 de mayo de 2000). Ley Orgánica de Educación Superior. Regitro Oficial \#77

Consejo de Educación Superior. (2015). Recuperado de http://www.CES.gob.ec

Consejo Nacional de Universidades y Escuelas Politécnicas. (1992). Evaluación de la situación actual y perspectivas para el corto y mediano plazos de las universidades y escuelas politecnicas: resumen del informe. Quito: Autor.

Galarza, G. (2004). La educación superior no universitaria en el Ecuador. Caracas: UNESCO/IESALC.

Ganga, F. A. (2005). Análisis preliminar del gobierno universitario chileno. Revista Venezolana de Gerencia, $10,213-246$

Levy, D. (1979). El gobierno de las universidades en Mexico desde una perspectiva internacional. Foro Internacional, 576-599.

Levy, D. (1979). Review: universities and governments: the comparative politics of higher education. Comparative Politics, 12(1), 99-121.

Levy, D. (2006). The unanticipated explosion: private higher education's global surge. Comparative Education Review, 50(2), 217-240.

Machasilla, E. P., Sánchez, D. y Urgilés, O. E. (2014). Repositorio de la Escuela Superior Politécnica del Litoral. Recuperado de http://www.dspace.espol.edu.ec

Minteguiaga, A. (2010). Los vaivenes en la regulación y evaluación de la educación superior en Ecuador. El Caso del Mandato 14 en el Contexto Constituyente. En R. Ramirez (Ed.), Transformar a la Universidad para Transformar a la Sociedad (pp. 83-123). Quito: SENPLADES.

Organización de Estados Iberoamericanos para la Educación, la Ciencia y la Cultura. (2013). Miradas sobre la educación en Iberoamérica. Desarrollo profesional docente y mejora de la educacion. Madrid: Autor.

Pacheco, L. (2008). La Universidad. Desafíos en la gestión académica. Recuperado de http://www.lucaspachecoprado.com/sites/default/files/documentos/la universidad desafios en la gesti on academica.pdf

Pacheco, L. (2011). Evolución histórica de la universidad en el Ecuador: 1603-2010. En Simposio Permanente sobre la Universidad (pp. 11-36). Pontificia Universidad Católica del Ecuador.

Post, D. (2011). Las reformas constitucionales en el Ecuador y las oportunidades para el acceso a la educación superior desde 1950. Archivos Analíticos de Políticas Educativas, 19(20), 1-24. Recuperado de http://epaa.asu.edu/ojs/article/view/814/922

Ramírez, R. (2010). Introducción. En SENPLADES, Transformar a la Universidad para Transformar a la Sociedad. (pp. 7-26). Quito. 
Ramírez, R. (2010). Justicia distributiva en la universidad ecuatoriana, 1996-2006 (disputa teórico/práctica y política de la gratuidad de la educación universitaria). Transformar la Universidad para Transformar la Sociedad. Secretaría Nacional de Planificación y Desarrollo.

Ramírez, R. (29 de 05 de 2013). Tercera ola de transformación de la educación superior en Ecuador. Hacia la constitucionalización de la sociedad del buen vivir. Quito: SENESCYT.

Schwartzman, S. y Klein, L. (1994). Higher Education and government in Brazil. Recuperado de http://www.schwartzman.org.br/simon/vught.htm

SENESCYT. (2013). Directorio de Universidades y Escuelas Politécnicas: Listado de ubicaciones matrices y sedes. Ecuador: Autor.

Sistema Nacional de Información. (2014). Sistema Nacional de Información. Recuperado de http://indestadistica.sni.gob.ec/QvAJAXZfc/opendoc.htm?document=SNI.qvw\&host=QVS@kukuri\&anony mous=truehttp://indestadistica.sni.qob.ec/QvAJAXZfc/opendoc.htm?document=SNI.qvw\&host=QVS@ku kuri\&anonymous=true\&bookmark=Document/BM37

Villavicencio, A. (2012). Evaluación y acreditación en tiempos de cambio: La política pública universitaria en cuestionamiento. Quito: IAEN.

Weise, C. y Laguna, J. L. (2009). Higher education in the andean countries: Bolivia, Peru and Ecuador. En F. L. Segrera, C. Brock y J. D. Sobrinho, Higher education in Latin America and the Caribbean. UNESCO/IESALC. 